\title{
Graft-versus-host Disease After Double-Unit Cord Blood Transplantation Has Unique Features and an Association with Engrafting Unit-Recipient HLA-match
}

\author{
D. M. Ponce ${ }^{1,3}$, A. Gonzales ${ }^{1}$, M. Lubin ${ }^{1}$, H. Castro-Malaspina ${ }^{1,3}$, S. Giralt ${ }^{1,3}$, J. D. \\ Goldberg $^{1,3}$, A. M. Hanash ${ }^{1}$, A. Jakubowski ${ }^{1,3}$, R. Jenq ${ }^{1,3}$, E. B. Papadopoulos ${ }^{1,3}$, M. A. \\ Perales $^{1,3}$, M.R.M. van den Brink ${ }^{1,3}$, J. W. Young ${ }^{1,3}$, F. Boulad ${ }^{2}$, R. J. O'Reilly ${ }^{2}$, S. Prockop ${ }^{2}$, \\ T.N. Small ${ }^{2}$, A. Scaradavou ${ }^{2}$, N. A. Kernan ${ }^{2}$, C. E. Stevens ${ }^{1}$, and J. N. Barker ${ }^{1,3}$ \\ ${ }^{1}$ Adult Bone Marrow Transplantation Service, Department of Medicine, Memorial Sloan-Kettering \\ Cancer Center, New York, New York \\ ${ }^{2}$ Bone Marrow Transplantation Service, Department of Pediatrics, Memorial Sloan-Kettering \\ Cancer Center, New York, New York \\ ${ }^{3}$ Weill Cornell Medical College, New York, New York
}

\begin{abstract}
Manifestations and risk factors of graft-versus-host disease (GVHD) after double-unit cord blood transplantation (DCBT) are not firmly established. We evaluated 115 DCBT recipients (median age 37 years) transplanted for hematologic malignancies with myeloablative or non-myeloablative conditioning and calcineurin-inhibitor/ mycophenolate mofetil immunosuppression. Incidences of day 180 grade II-IV and III-IV acute GVHD (aGVHD) were 53\% (95\%CI: 44-62) and 23\% (95\%CI: 15-31), respectively, with a median onset of 40 days (range 14-169). Eighty percent of patients with grade II-IV aGVHD had gut involvement, and $79 \%$ and $85 \%$ had day 28 treatment responses to systemic corticosteroids or budesonide, respectively. Of 89 engrafted patients cancerfree at day 100, 54\% subsequently had active GVHD with $79 \%$ of those affected having persistent or recurrent aGVHD or overlap syndrome, whereas late GVHD in the form of classical chronic GVHD was uncommon. Notably, grade III-IV aGVHD incidence was lower if the engrafting unit human leukocyte antigen (HLA)-A,-B,-DRB1 allele match was $>4 / 6$ to the recipient (HR 0.385, p $=0.031$ ), whereas engrafting unit infused nucleated cell dose and unit-unit HLA-match were not significant. GVHD after DCBT was common in our study, predominantly affected the gut, had a high therapy response, and late GVHD frequently had acute features. Our findings support the consideration of HLA- A,-B,-DRB1 allele donor-recipient (but not unit-unit) HLA-match in unit selection, a practice change in the field. Moreover, new prophylaxis strategies that target the gastrointestinal tract are needed.
\end{abstract}

\footnotetext{
(C) 2013 The American Society for Blood and Marrow Transplantation. Published by Elsevier Inc. All rights reserved. Corresponding Author: Juliet N. Barker, Box 259, 1275 York Ave, NY, NY, 10065, Ph 212 639 3468, Fax 2127173500 , barkerj@mskcc.org.

Author Contributions: D.M.P. and C.E.S. interpreted the data and wrote the manuscript. M.L. and A.G. analyzed the data and wrote the manuscript. H.C.M., S.G., J.D.G., A.M.H., A.J., E.P., M.A.P., M.R.M.v.B., J.W.Y., F.B, R.O.R., S.P., T.S., A.S., and N.A.K. wrote the manuscript. J.N.B. designed the study, interpreted the data, and wrote the manuscript.

Conflict of Interest: The authors have no relevant conflicts of interest to declare.

Publisher's Disclaimer: This is a PDF file of an unedited manuscript that has been accepted for publication. As a service to our customers we are providing this early version of the manuscript. The manuscript will undergo copyediting, typesetting, and review of the resulting proof before it is published in its final citable form. Please note that during the production process errors may be discovered which could affect the content, and all legal disclaimers that apply to the journal pertain.
} 


\section{Introduction}

In recent years the use of cord blood (CB) as an alternative hematopoietic stem cell (HSC) source has increased substantially, especially using double-unit grafts for larger children and adults. However, while the incidence of graft-versus-host disease (GVHD) is low given the marked degree of human leukocyte antigen (HLA)-mismatch of CB grafts ${ }^{1,2}$, GVHD remains a burden after $\mathrm{CB}$ transplantation (CBT). This is especially true if patients are transplanted without anti-thymocyte globulin (ATG) ${ }^{3,4}$. Furthermore, although progressionfree survival (PFS) after double-unit CBT (DCBT) is comparable to that of adult donor transplantation ${ }^{4-6}$, DCBT may be associated with an increased incidence of acute GVHD (aGVHD) when compared to single-unit $\mathrm{CBT}^{3}$. While Macmillan et al suggested that only grade II aGVHD is increased after DCBT $^{3}$, GVHD has emerged as a major cause of morbidity and mortality after $\mathrm{DCBT}^{4}$, and data concerning its manifestations, treatment response, and risk factors are limited. Moreover, the incidence and clinical characteristics of GVHD after day 100 in DCBT recipients have not been well described. We, therefore, investigated the incidence, nature, and treatment response of aGVHD, the risk factors associated with severe aGVHD, and the manifestations of chronic GVHD (cGVHD) in 115 pediatric and adult DCBT recipients with hematologic malignancies transplanted with a calcineurin- inhibitor/ mycophenolate mofetil (MMF) and no ATG.

\section{Patients and Methods}

\section{Patient and Graft Characteristics}

This analysis was performed in patients transplanted at Memorial Sloan-Kettering Cancer Center (MSKCC) between October 1, 2005 and February 28, 2011. All CBT recipients during this time period received double-unit grafts. Patients eligible for this analysis included all consecutive adult and pediatric DCBT recipients of first allograft transplanted for the treatment of hematologic malignancies excluding acute leukemia patients transplanted with $>20 \%$ bone marrow blasts. All patients provided written informed consent for transplantation according to the principles of the Declaration of Helsinki, and transplantation outcome analysis was approved by the MSKCC Institutional Review and Privacy Board.

CB units were selected on the basis of 4-6/6 HLA-A,-B antigen, -DRB1 allele match to the recipient, a cryopreserved total nucleated cell (TNC) dose of at least $1.5 \times 10^{7} /$ kilogram $(\mathrm{kg}) /$ unit, and the bank of origin as previously described ${ }^{7}$. Unit-unit HLA-match was not considered in CB unit selection. High resolution HLA-A,-B,-C,-DRB1 and DQ allele typing of $\mathrm{CB}$ units was performed routinely but usually did not influence unit selection. Units were thawed using albumin-dextran dilution ${ }^{8}(\mathrm{n}=205)$ or thawed with wash $(\mathrm{n}=25)$.

\section{Conditioning Regimens and GVHD Prophylaxis and Treatment}

All patients were hospitalized in high-efficiency particulate air filtered rooms and received similar supportive care. Pre-transplant conditioning varied according to patient's age, diagnosis, remission status, extent of prior therapy, and co-morbidities, and consisted of myeloablative (high-dose and reduced intensity), and non-myeloablative regimens (Table 1). Granulocyte-colony-stimulating factor $(5 \mathrm{mcg} / \mathrm{kg} /$ day $)$ was given to all patients after transplant until neutrophil recovery.

All patients received a calcineurin-inhibitor and MMF for GVHD prophylaxis starting day -3 , and none received $\mathrm{ATG}^{4,9}$. One-hundred and four patients received cyclosporine-A targeting a trough level of $200-400 \mathrm{ng} / \mathrm{ml}$, and eleven patients received tacrolimus targeting a trough level of 5-15 ng/ml. In the first 83 patients MMF was dosed at 1 gram (or $15 \mathrm{mg} / \mathrm{kg}$ if $<50 \mathrm{~kg}$ ) every 12 hours; the total daily dose was increased ( $1 \mathrm{gram} / \mathrm{dose}$ if $>50 \mathrm{~kg}, 15$ 
$\mathrm{mg} / \mathrm{kg} / \mathrm{dose}$ if $<50 \mathrm{~kg}$ and $>12$ years, and $20 \mathrm{mg} / \mathrm{kg} /$ dose to a maximum of 1 gram if $<12$ years, all every 8 hours) in the subsequent 32 patients to augment GVHD prophylaxis ${ }^{10}$. MMF was initiated intravenously in all patients and this was maintained during hospitalization and switched to oral prior to discharge. In the absence of GVHD, MMF was tapered after day 45, and the calcineurin-inhibitor was tapered after day 100. Patients with grade II-IV aGVHD were initially treated with either single-agent budesonide ( $9 \mathrm{mg}$ daily) or systemic corticosteroids (1-2 $\mathrm{mg} / \mathrm{kg}$ oral prednisone or intravenous methylprednisolone) at the treating physician's discretion according to the clinical GVHD severity at the time of diagnosis.

\section{Study Definitions}

Disease risk, time to neutrophil and platelet recovery, and sustained donor engraftment were defined as previously described ${ }^{4,11}$. Patient ancestry was classified as European when there was no known non-European ancestry. Non-Europeans were Asian, African, white Hispanic, and Middle Eastern patients as well as those with mixed non-European and European origins ${ }^{12}$. Donor chimerism was determined serially in the bone marrow and blood using semi-quantitative analysis of polymerase chain reaction amplified informative polymorphisms ${ }^{11,13}$.

aGVHD and cGVHD were diagnosed clinically with histological confirmation as required and clinically appropriate. Grade of aGVHD was based on the International Bone Marrow Transplant Registry classification ${ }^{14}$ except grades A-D were labeled grades I-IV and was applied to GVHD with purely acute features even if it occurred after day 100. Grading was reviewed by a transplant clinician panel to reach consensus of maximum aGVHD grade. cGVHD was defined according to published National Institutes of Health consensus criteria $^{15}$, and these criteria were used to assess GVHD severity for classical and overlap cGVHD. Malignant relapse was defined as recurrence or progression of disease over pretransplant baseline, and TRM as death from any cause in continued remission. PFS was defined according to standard criteria. The algorithm of Copelan et al was used to assign the primary cause of death ${ }^{16}$.

Responses to aGVHD therapy were determined using published definitions ${ }^{3}$. Day 28 was chosen as the optimal time-point to assess GVHD response ${ }^{17,18}$. Complete response was the complete resolution of aGVHD symptoms in all organs without secondary GVHD therapy. Partial response was the improvement in GVHD stage in all initially affected organs without complete resolution, and without worsening in other GVHD target organs or requiring secondary GVHD therapy. Progression was worsening of GVHD in at least 1 organ with or without amelioration in any organ. No response was patients with the same grade of GVHD not meeting criteria for partial response or progression.

\section{Statistical Analysis}

Data on patient characteristics and transplant-related outcomes were obtained from the prospectively maintained MSKCC transplant database verified by primary source documentation. Significant differences in categorical variables were determined by $\mathrm{Chi}$ square or Fisher's exact test (two-tailed). Significant differences between means were determined by Students $t$ test and between distributions by the Mann-Whitney U test. Incidences of neutrophil engraftment, GVHD and relapse were estimated using the cumulative incidence function. Death was the competing event for engraftment and relapse. Relapse and death were the competing events for GVHD. We included relapse as a competing event for GVHD calculations because treatment of relapse could alter GVHD incidence and severity. PFS was estimated using Kaplan- Meier methodology. Univariate and multivariate Cox regression analyses were used to ascertain associations between patient 
or graft characteristics with severe (grade III-IV) aGVHD. Analyses were performed using SPSS version 19.

\section{Results}

\section{Characteristics of Patients and Grafts}

Patient demographics are summarized in Table 1. One-hundred and fifteen patients, predominantly adults (median age 37 years, 23 children and 92 adults) were transplanted. More than half had non-European ancestry. The majority had high-risk acute leukemia and received high dose or reduced intensity conditioning. TNC, CD34+ and CD3+ cell doses, and HLA unit-recipient and unit-unit match are shown in Table 2. Unit selection was based on match at HLA-A,-B antigens and -DRB1 alleles with up to 2 mismatches allowed. However, high resolution typing of HLA-A,-B along with -DRB1 alleles demonstrated 87 units $(38 \%)$ were $<4 / 6$ HLA-allele matched to the recipient. The median match grade of units at 6 HLA-alleles (high resolution match grade) was $4 / 6$ (range 1-6/6), and for 10 alleles (including HLA-C and -DQ) was 6/10 (range 2-9/10). Since unit-unit match was not a selection criterion, there was a broad range of unit-unit match at all levels of resolution of HLA typing (Table 2).

\section{Engraftment and Donor Chimerism}

Six patients had graft failure (5 primary, 1 secondary), and one patient was not evaluable due to early death. The cumulative incidence of sustained donor-derived neutrophil engraftment was $94 \%$ (95\%CI: 90-98) by day 45, and platelet engraftment was $84 \%$ (95\%CI: 78-91) by day 180 . The median times to neutrophil and platelet recovery were 24 days (range 12-43) and 50 days (range 29-162), respectively, after myeloablative conditioning, and 10 days (range 7-36) and 34 days (range 9-59), respectively, after nonmyeloablative conditioning.

One unit dominated in all engrafting patients. Eighty-nine (82\%) of the 108 patients with sustained donor engraftment had complete dominance of a single unit documented as early as 21-28 days after transplantation (median donor engraftment: 100\%). The percentage of patients with hematopoiesis entirely derived from a single unit further increased to 92 (91\%) of 101 evaluable patients at day 60, and 84 (94\%) of 89 evaluable patients at day 100 (median 100\% donor with the engrafting unit at both time points). The HLA-match of the engrafting unit with the recipient was a median of 4/6 HLA-A,-B,-DRB1 alleles (range 16/6), and 6/10 HLA-A,-B,-C,-DRB1,-DQ alleles (range 2-9/10).

\section{Acute GVHD Incidence and Manifestations}

Sixty-one patients had grade II-IV aGVHD within the first 6 months after DCBT (2 with late onset after day 100). The diagnosis was supported by biopsy in 55/61 (90\%) of patients [skin $(\mathrm{n}=29)$, GI tract $(\mathrm{n}=44)$, and liver $(\mathrm{n}=5)$ ]. Twenty-six of these patients had grade III-IV disease (with 3 peaking at grade III-IV after day 100). The day 180 cumulative incidences of grade II-IV and III-IV aGVHD were 53\% (95\% CI: 44-62) and 23\% (95\% CI: 15-31), respectively (Figure 1). Among patients with grade II-IV aGVHD, the median onset of aGVHD was 40 days (range 14-169); it was earlier for those with grade III-IV disease (median 35 days) than those with grade II aGVHD (median 42 days, $\mathrm{p}=0.012$ ).

Of patients with grade II-IV aGVHD, the gastro-intestinal (GI) tract was the most commonly affected organ ( $n=49,80 \%$ of those with grade II-IV disease). Fourteen patients had upper GI tract involvement, 9 had lower, and 26 had both. Skin was affected less often $(\mathrm{n}=39,64 \%$ of those with grade II-IV disease). Only 17 patients had stage III skin disease, and 2 had stage IV. The liver was involved in $18 \%$ of grade II-IV cases. The combination of 
skin and GI tract disease was the most common manifestation $(n=25,41 \%$ of those affected), followed by GI tract alone (25\%), and skin alone (16\%). Four patients had involvement of all 3 organs, 5 had gut and liver involvement, and 2 had only liver involvement.

\section{Acute GVHD Treatment and Responses}

Among patients with grade II-IV aGVHD, 29 (48\%) were treated with systemic corticosteroids, 27 (44\%) with budesonide alone, 4 (7\%) with topical corticosteroids, and one recovered without additional immunosuppression. Most patients who received systemic corticosteroids had grade III-IV disease and the majority (79\%) responded by day 28 of therapy (Table 3). Six patients (4 adults, 2 children) did not respond, and 2 that initially responded had subsequent progression of GI aGVHD.

Budesonide was used as the sole treatment exclusively in adults for overall grade II aGVHD predominantly affecting the upper and/or lower GI tract. Compared with those given systemic corticosteroids, these patients had a later GVHD onset (Table 3). Twenty-three ( $85 \%$ ) of the budesonide-treated patients responded by day 28 of therapy. Four patients without an initial response also required salvage with systemic corticosteroids and 3 responded.

\section{GVHD after Day 100: Late Acute and Chronic GVHD}

As day 100 has traditionally distinguished acute from chronic GVHD, the manifestations of late GVHD occurring after day 100 were examined (Table 4). Of the 89 patients who engrafted and were in remission at day 100, 41 had no GVHD after day 100. Seventeen of these 41 patients ( $42 \%$ ) had had prior aGVHD (12 grade II and 5 grade III) that had resolved. The remaining 48 patients had active GVHD on or beyond day 100. Twenty-nine of these patients had persistent $(n=14)$ or recurrent $(n=15)$ aGVHD (3 subsequently evolved into overlap syndrome), and 2 had late onset aGVHD on days 161 and 169. An additional 17 patients developed cGVHD after day 100. Ten had classical cGVHD (4 interrupted onset, 6 de novo), and 7 had overlap syndrome (6 with prior aGVHD and one de novo).

Thus, of the 48 patients with active GVHD after day 100, $38(79 \%)$ had purely acute features or overlap syndrome. Overall, among patients who engrafted and were cancer-free as of day 100, the 2-year cumulative incidence of GVHD of any kind was 54\% (95\%CI: 4465) (Figure 2A). However, if only chronic GVHD by NIH criteria was considered, the 2year incidence was 23\% (95\%CI: 14-32) (Figure 2B).

Disease manifestations were examined in the 20 patients who developed NIH defined ${ }^{15}$ cGVHD (including the 3 patients who evolved from aGVHD to overlap syndrome). The 10 patients with classical cGVHD had a median onset of 233 days (range 141-505) with skin pigment changes $(n=5)$, dry eyes $(n=3)$, joint pain $(n=1)$, anorexia $(n=2)$, liver function test abnormalities $(n=4)$, and nephrotic syndrome $(n=1)$. The 10 patients with overlap syndrome (median onset 156 days, range 100-418) had aGVHD symptoms of erythematous skin rash $(n=5)$, nausea/ vomiting and/ or diarrhea $(n=10)$ combined with dry mouth or taste alteration $(n=3)$, mouth ulcers $(n=1)$, dry eyes $(n=3)$, joint stiffness $(n=1)$, vaginal dryness $(n=1)$, and/or liver function test abnormalities $(n=1)$. No patient had severe ocular or sclerotic skin involvement, contractures, or symptomatic pulmonary involvement. The majority had disease of mild $(n=10,50 \%)$ or moderate $(n=5,25 \%)$ severity, and the GI tract and skin were the organs most commonly affected. Five patients $(25 \%)$ developed severe disease. All but 4 patients who developed cGVHD during the study period had done so by one year after transplant. 


\section{Cessation of Immunosuppression}

Sixty-eight patients had sustained donor-derived engraftment and were in remission at one year after DCBT. Of those, $24(35 \%)$ had stopped all immunosuppression (14 patients without GVHD and 10 with prior GVHD) whereas 44 (65\%) patients remained on immunosuppression (18 for active GVHD and 26 on a taper) at one year after DCBT. Of the 18 with active GVHD at one year, 10 had aGVHD, 5 had overlap syndrome, and 3 had classical cGVHD.

\section{Mortality and GVHD Deaths}

A total of 44 patients have died to date; 14 from relapse, 29 from transplant-related complications, and one patient who was transplanted for treatment-related acute myeloid leukemia from relapsed osteosarcoma. With a median follow-up of 33 months (range 8-73), the 2-year overall survival and PFS are 66\% (95\%CI: 57-75) and 60\% (95\%CI: 51-70), respectively.

GVHD was the primary cause of death in 9 patients and was the second most common cause of TRM after organ toxicity. These deaths occurred at a median of 184 days (range 70-445) after DCBT. The 9 GVHD deaths were in adults and due to acute disease (1 grade II, 7 grade III-IV) or grade III aGVHD evolving to severe overlap syndrome $(\mathrm{n}=1)$. Five of these patients had initially received systemic corticosteroids and 4 had failed initial treatment with budesonide. The most common secondary cause of death was infection ( $\mathrm{n}=$ 7). Two had pneumonia (presumed bacterial), 3 had proven bacterial infections ( 1 fulminant C. Difficile colitis, 1 Klebsiella septicemia, and 1 Klebsiella pneumonia), and 2 had disseminated adenovirus. The remaining patients died of GI tract perforation $(\mathrm{n}=1)$ and idiopathic diffuse alveolar hemorrhage $(\mathrm{n}=1)$.

\section{Risk Factors for Grade III-IV aGVHD}

As the predominant GVHD syndrome was acute disease, and grade III-IV disease accounted for nearly all GVHD mortality, we evaluated risk factors for grade III-IV aGVHD through day 180 (to account for patients whose aGVHD peaked in severity after day 100). Univariate analysis of the relationship between patient characteristics and day 180 grade III-IV aGVHD revealed no association with patient age ( $0-15$ vs $>16$ years), diagnosis (acute leukemia/ MDS vs lymphoma/ CLL), conditioning intensity (myeloablative vs nonmyeloablative), patient ancestry (European vs non-European), recipient CMV serostatus (sero-negative vs sero-positive recipient), or MMF dosing interval (every 12 vs every 8 hours).

In univariate analysis, there was no association with the infused TNC doses/kg or the CD3+ cell doses $/ \mathrm{kg}$ of either the engrafting unit or the total dose (unit \#1 + unit \#2) in the graft. $\mathrm{CD} 34+$ cell doses also had no relationship with severe aGVHD risk. There was no association between the unit-unit HLA-match (at any degree of resolution) and severe aGVHD. Differences in severe aGVHD incidence according to HLA-match of the engrafting unit to the recipient (as shown in Figure 3) did not reach significance.

A multivariate Cox regression analysis was performed to take potential confounding variables into account (Table 5). In this analysis, better 6 allele HLA-match between the engrafting unit and the recipient was associated with a lower incidence of severe aGVHD (p $=0.031$ ). Patient age, recipient CMV serostatus, engrafting unit infused TNC, and unit-unit HLA-match were not significant. When 10 allele HLA-match of the engrafting unit was substituted for a 6 allele match in the multivariate analysis, the severe aGVHD hazard ratio was 0.325 for engrafting units that were $>8 / 10$ HLA-matched to the recipient but the comparison was no longer significant $(\mathrm{p}=0.073)$. 


\section{Discussion}

The clinical features and risk factors of GVHD after DCBT are yet to be firmly established. Our study is the first to examine GVHD using a pediatric and adult population uniformly transplanted with double-unit CB grafts, calcineurin inhibitor plus MMF prophylaxis, and no ATG. Whether double-unit grafts increase GVHD risk over that of singles could not be examined since we have not performed single-unit CBT. This will be addressed, at least in children, in the Blood and Marrow Transplant Clinical Trials Network randomized single versus double-unit pediatric CBT trial. However, we confirmed that over half of DCBT recipients are affected by clinically significant aGVHD. Furthermore, while our day 180 incidences of grades II-IV and III-IV aGVHD of 53\% and 24\% were similar to the 58\% and $19 \%$ day 100 incidences reported by Macmillan et $\mathrm{al}^{3}$, we found a greater proportion of GI tract as opposed to the predominantly skin involvement reported in that series. Interestingly, Alsultan et al. have also reported in an analysis of 79 pediatric CBT recipients that the organ most commonly affected was the GI tract ${ }^{19}$. Differences in patient populations, aggressive efforts to diagnose aGVHD with early endoscopic evaluation and biopsy, and our uniform lack of ATG use could have contributed to these findings. The striking propensity for GI involvement in our study suggests that GVHD prophylaxis targeting the gut could be a promising strategy with agents such as inhibitors of lymphocyte migration to the GI tract $^{20-23}$, disruption of the IL-21 pathway ${ }^{24-26}$, JAK2 inhibition ${ }^{27}$, or agents promoting IL-22 expression ${ }^{28}$.

While our study has the limitations of a retrospective series, our aGVHD treatment responses compare favorably with those after related and unrelated donor HSC transplantation ${ }^{17}$. Enhanced response to therapy has been described with cGVHD after $\mathrm{CBT}^{29}$, and future prospective comparisons of aGVHD therapy responses after the transplantation of $\mathrm{CB}$ versus adult donors will be of great interest. In addition, many patients with grade II gut aGVHD were successfully treated with single agent budesonide. The poorly absorbed corticosteroids budesonide and beclomethasone (reviewed by Ibrahim et $\mathrm{al}^{30}$ ) have reported activity in GVHD therapy after adult donor allografts ${ }^{31,32}$. However, we did not determine the extent of systemic absorption. In DCBT recipients, systemic budesonide exposure could be enhanced by cytochrome P4503A4 inhibition by azoles (as described with inhaled budesonide ${ }^{33}$ ) in which case budesonide may be no different to low dose oral prednisone. Overall, our data suggest that budesonide may have activity in grade II GI GVHD in CBT recipients. While not all patients responded, and lack of prompt response should trigger immediate initiation of systemic corticosteroids, our data suggest prospective clinical trials of budesonide therapy in CBT recipients with grade II disease are warranted with the aim to potentially decrease systemic corticosteroid exposure but with careful determination of the extent of systemic absorption.

A further unique finding of this analysis was that $79 \%$ of patients with active GVHD after day 100 had acute features with persistent, recurrent, or late aGVHD, or overlap syndrome, and the disease predominantly affected the gut. Classical cGVHD affected only 10 patients in the study, and cGVHD was usually of mild or moderate severity. Only one patient had ulceration of the oral mucosa, and no patients had moderate or severe ocular GVHD, contractures, or pulmonary involvement. By contrast, Arai et al reported that the skin, lung and eye scores determined the severity of cGVHD in a 298 patient series of predominantly related and unrelated donor peripheral blood HSC transplantation recipients ${ }^{34}$. Thus, the pathophysiology of the late GVHD in most DCBT recipients appears distinct from that of classical cGVHD after transplantation of adult peripheral blood HSC. Overall, while classical cGVHD was uncommon, when all patients with active GVHD (II-IV aGVHD, overlap GVHD, and classical cGVHD) were combined, this accounted for the high 54\% incidence of active GVHD in day 100 survivors in our study as compared to other CBT 
series ${ }^{1,2,35-37}$. Such an analysis is the only way to reflect the true burden of late GVHD in allograft survivors.

The rates of grade III-IV aGVHD in this study, while no worse than those observed after unmodified unrelated donor peripheral blood stem cell transplantation ${ }^{38}$, emphasize the need for enhanced GVHD prophylaxis after DCBT. Omission of ATG in the conditioning regimen has been identified as an aGVHD risk factor $^{3}$, and some centers have elected to include ATG with consequent low rates of GVHD 6,39 . However, ATG has been associated with an increased risk of serious infections including lethal Epstein-Barr virus lymphoproliferative disease $e^{40,41}$, delayed immune reconstitution ${ }^{42}$, and increased risk of transplant-related mortality ${ }^{43}$. ATG can also increase the risk of relapse after reduced intensity allograft ${ }^{44}$. Therefore, other approaches to prevent and treat GVHD are more appealing, especially as robust T-cell recovery is observed after DCBT in the absence of ATG $^{9}$. During this study we increased the MMF dose to augment GVHD prophylaxis. More recently, as a result of this analysis, we have also delayed the commencement of the MMF taper. While future studies will analyze the impact of these interventions, ultimately improved understanding of the distinct pathophysiology of GVHD after DCBT is needed to facilitate improved prevention and treatment of this disease. Use of biomarkers to predict subsequent severe pathology or treatment outcomes could also be of use as has been described in adult donor $\mathrm{HSCT}^{45-47}$.

It is notable that just as the CD34+ cell dose $/ \mathrm{kg}$ of the engrafting unit determines the speed and success of engraftment after DCBT ${ }^{11}$, our data suggests its HLA-match to the patient at 6 alleles influences aGVHD severity. Delaney et al have previously reported a trend toward a lower risk of aGVHD if units of a double-unit CB graft were HLA-DR matched to the recipient in $22 \mathrm{DCBT}$ recipients transplanted with $\mathrm{CSA} / \mathrm{MMF}^{48}$. Prioritizing high-resolution donor-recipient matching in unit selection could, therefore, provide another strategy to lower the incidence of severe aGVHD. In multivariate analysis, we found a greater significance with 6 versus 10 allele engrafting unit-recipient HLA-match. Although analysis of larger numbers of patients is required for definitive conclusions, this suggests that only 6 allele match may be sufficient to facilitate a reduction in severe aGVHD. In this study, 36 patients engrafted with a unit that was 1-3/6 HLA-A,-B,-DRB1 allele matched. It is possible that some may have had a better matched unit available of similar cell dose if high resolution matching of HLA-A and -B had been employed. Six allele matching would be relatively easy to implement in smaller children with units $>5.0 \times 10^{7} \mathrm{TNC} / \mathrm{kg}$ above which further dose increments have not been shown to be beneficial in single-unit $\mathrm{CBT}^{37}$, and the same is likely to be true in DCBT. Such matching criteria may be more challenging in larger patients, however, and further analyses will be required to determine the dose threshold above which high-resolution HLA-match could safely be given priority. Another finding of practical significance was that there was no advantage associated with HLA-match of the units to each other, as we have also previously reported with engraftment ${ }^{11}$. While continued study is appropriate, no data supports consideration of unit-unit match in CB selection from the standpoint of engraftment or aGVHD at this time.

Despite the incidence of grade III-IV aGVHD we found a relatively high survival after DCBT overall. This was attributable to the low late mortality and protection against relapse as previously reported by our group ${ }^{4}$ and others ${ }^{5}$. Nonetheless, even though no children have died from GVHD, the morbidity of severe disease has been considerable in some pediatric and adult DCBT recipients, and GVHD has been the second most common cause of TRM affecting 9 adults in our study. This highlights the need for improved prophylaxis and therapy. Furthermore, although one third of patients came off immunosuppression by one year after DCBT, the speed of GVHD resolution, the time to cessation of 
immunosuppression, and patient quality of life after CBT compared with adult donor transplantation should be explored in future studies.

\section{Acknowledgments}

This work was supported in part by the Gabrielle's Angel Foundation for Cancer Research (J.N.B.), the Memorial Sloan-Kettering Cancer Center Society (J.N.B.), the Translational and Integrative Medicine Research Grant (J.N.B.), P01 CA23766 from the National Cancer Institute, National Institutes of Health (J.N.B.), the by the New York State Empire Clinical Research Investigator Program (D.M.P.), and the Society of Memorial Sloan-Kettering Cancer Center.

\section{References}

1. Eapen M, Rubinstein P, Zhang MJ, et al. Comparison of outcomes after transplantation of unrelated donor umbilical cord blood and bone marrow in children with acute leukemia. Lancet. 2007; 369:1947-1954. [PubMed: 17560447]

2. Eapen M, Rocha V, Sanz G, et al. Effect of graft source on unrelated donor haemopoietic stem-cell transplantation in adults with acute leukaemia: a retrospective analysis. Lancet Oncol. 2010; 11:653-660. [PubMed: 20558104]

3. MacMillan ML, Weisdorf DJ, Brunstein CG, et al. Acute graft-versus-host disease after unrelated donor umbilical cord blood transplantation: analysis of risk factors. Blood. 2009; 113:2410-2415. [PubMed: 18997171]

4. Ponce DM, Zheng J, Gonzales AM, et al. Reduced late mortality risk contributes to similar survival after double-unit cord blood transplantation compared with related and unrelated donor hematopoietic stem cell transplantation. Biol Blood Marrow Transplant. 2011; 17:1316-1326. [PubMed: 21232625]

5. Brunstein CG, Gutman JA, Weisdorf DJ, et al. Allogeneic hematopoietic cell transplantation for hematological malignancy: relative risks and benefits of double umbilical cord blood. Blood. 2010; 116:4693-4699. [PubMed: 20686119]

6. Chen YB, Aldridge J, Kim HT, et al. Reduced-intensity conditioning stem cell transplantation: comparison of double umbilical cord blood and unrelated donor grafts. Biol Blood Marrow Transplant. 2012; 18:805-812. [PubMed: 22015993]

7. Barker JN, Byam C, Scaradavou A. How I treat: the selection and acquisition of unrelated cord blood grafts. Blood. 2011; 117:2332-2339. [PubMed: 21149636]

8. Barker JN, Abboud M, Rice RD, et al. A "no-wash" albumin-dextran dilution strategy for cord blood unit thaw: high rate of engraftment and a low incidence of serious infusion reactions. Biol Blood Marrow Transplant. 2009; 15:1596-1602. [PubMed: 19896084]

9. Sauter C, Abboud M, Jia X, et al. Serious infection risk and immune recovery after double-unit cord blood transplantation without antithymocyte globulin. Biol Blood Marrow Transplant. 2011; 17:1460-1471. [PubMed: 21310254]

10. Jacobson PA, Huang J, Wu J, et al. Mycophenolate pharmacokinetics and association with response to acute graft-versus-host disease treatment from the Blood and Marrow Transplant Clinical Trials Network. Biol Blood Marrow Transplant. 2010; 16:421-429. [PubMed: 19925875]

11. Avery S, Shi W, Lubin M, et al. Influence of infused cell dose and HLA-match on engraftment after double-unit cord blood allografts. Blood. 2011; 117:3277-3285. [PubMed: 21149633]

12. Barker JN, Byam CE, Kernan NA, et al. Availability of cord blood extends allogeneic hematopoietic stem cell transplant access to racial and ethnic minorities. Biol Blood Marrow Transplant. 2010; 16:1541-1548. [PubMed: 20800103]

13. Avery S, Voss MH, Gonzales AM, et al. Importance of day 21 BM chimerism in sustained neutrophil engraftment following double-unit cord blood transplantation. Bone Marrow Transplant. 2011; 47(8):1056-1060. [PubMed: 22139066]

14. Rowlings PA, Przepiorka D, Klein JP, et al. IBMTR Severity Index for grading acute graft-versushost disease: retrospective comparison with Glucksberg grade. British Journal of Haematology. 1997; 97:855-864. [PubMed: 9217189] 
15. Filipovich AH, Weisdorf D, Pavletic S, et al. National Institutes of Health consensus development project on criteria for clinical trials in chronic graft-versus-host disease: I. Diagnosis and staging working group report. Biol Blood Marrow Transplant. 2005; 11:945-956. [PubMed: 16338616]

16. Copelan E, Casper JT, Carter SL, et al. A scheme for defining cause of death and its application in the T cell depletion trial. Biol Blood Marrow Transplant. 2007; 13:1469-1476. [PubMed: 18022577]

17. MacMillan ML, Weisdorf DJ, Wagner JE, et al. Response of 443 patients to steroids as primary therapy for acute graft-versus-host disease: comparison of grading systems. Biol Blood Marrow Transplant. 2002; 8:387-394. [PubMed: 12171485]

18. Levine JE, Logan B, Wu J, et al. Graft-versus-host disease treatment: predictors of survival. Biol Blood Marrow Transplant. 2010; 16:1693-1699. [PubMed: 20541024]

19. Alsultan A, Giller RH, Gao D, et al. GVHD after unrelated cord blood transplant in children: characteristics, severity, risk factors and influence on outcome. Bone Marrow Transplant. 2011; 46:668-675. [PubMed: 20676147]

20. Petrovic A, Alpdogan O, Willis LM, et al. LPAM (alpha 4 beta 7 integrin) is an important homing integrin on alloreactive T cells in the development of intestinal graft-versus-host disease. Blood. 2004; 103:1542-1547. [PubMed: 14563643]

21. Wysocki CA, Panoskaltsis-Mortari A, Blazar BR, Serody JS. Leukocyte migration and graftversus-host disease. Blood. 2005; 105:4191-4199. [PubMed: 15701715]

22. Reshef R, Luger S, Hexner EO, et al. Blockade of lymphocyte chemotaxis in visceral graft-versushost disease. New Engl J Med. 2012; 367(2):135-145. [PubMed: 22784116]

23. Waldman E, Lu SX, Hubbard VM, et al. Absence of beta7 integrin results in less graft-versus-host disease because of decreased homing of alloreactive T cells to intestine. Blood. 2006; 107:17031711. [PubMed: 16291587]

24. Bucher C, Koch L, Vogtenhuber C, et al. IL-21 blockade reduces graft-versus-host disease mortality by supporting inducible T regulatory cell generation. Blood. 2009; 114:5375-5384. [PubMed: 19843883]

25. Hanash AM, Kappel LW, Yim NL, et al. Abrogation of donor T-cell IL-21 signaling leads to tissue-specific modulation of immunity and separation of GVHD from GVL. Blood. 2011; 118:446-455. [PubMed: 21596854]

26. Hippen KL, Bucher C, Schirm DK, et al. Blocking IL-21 signaling ameliorates xenogeneic GVHD induced by human lymphocytes. Blood. 2012; 119:619-628. [PubMed: 22077059]

27. Betts BC, Abdel-Wahab O, Curran SA, et al. Janus kinase-2 inhibition induces durable tolerance to alloantigen by human dendritic cell-stimulated $\mathrm{T}$ cells yet preserves immunity to recall antigen. Blood. 2011; 118:5330-5339. [PubMed: 21917753]

28. Hanash AM, Dudakov JA, Hua G, et al. IL-22 protects intestinal stem cells from immune mediated tissue damage and regulates sensitivity to graft vs. host disease. Immunity. 2012 (in press).

29. Arora M, Nagaraj S, Wagner JE, et al. Chronic graft versus host disease following unrelated donor hematopoietic stem cell transplantation: higher response rate in recipients of unrelated donor umbilical cord blood. Biol Blood Marrow Transplant. 2007; 13:1145-1152. [PubMed: 17889350]

30. Ibrahim RB, Abidi MH, Cronin SM, et al. Nonabsorbable corticosteroids use in the treatment of gastrointestinal graft-versus-host disease. Biol Blood Marrow Transplant. 2009; 15:395-405. [PubMed: 19285626]

31. Bertz H, Afting M, Kreisel W, Duffner U, Greinwald R, Finke J. Feasibility and response to budesonide as topical corticosteroid therapy for acute intestinal GVHD. Bone Marrow Transplant. 1999; 24:1185-1189. [PubMed: 10642806]

32. Andree H, Hilgendorf I, Leithaeuser M, et al. Enteral budesonide in treatment for mild and moderate gastrointestinal chronic GVHD. Bone Marrow Transplant. 2008; 42:541-546. [PubMed: 18641680]

33. Raaska K, Niemi M, Neuvonen M, Neuvonen PJ, Kivisto KT. Plasma concentrations of inhaled budesonide and its effects on plasma cortisol are increased by the cytochrome P4503A4 inhibitor itraconazole. Clin Pharmacol Ther. 2002; 72:362-369. [PubMed: 12386638] 
34. Arai S, Jagasia M, Storer B, et al. Global and organ-specific chronic graft-versus-host disease severity according to the 2005 NIH Consensus Criteria. Blood. 2011; 118:4242-4249. [PubMed: 21791424]

35. Wagner JE, Barker JN, DeFor TE, et al. Transplantation of unrelated donor umbilical cord blood in 102 patients with malignant and nonmalignant diseases: influence of CD34 cell dose and HLA disparity on treatment-related mortality and survival. Blood. 2002; 100:1611-1618. [PubMed: 12176879]

36. Laughlin MJ, Eapen M, Rubinstein P, et al. Outcomes after transplantation of cord blood or bone marrow from unrelated donors in adults with leukemia. N Engl J Med. 2004; 351:2265-2275. [PubMed: 15564543]

37. Barker JN, Scaradavou A, Stevens CE. Combined effect of total nucleated cell dose and HLAmatch on transplant outcome in 1061 cord blood recipients with hematological malignancies. Blood. 2010; 115:1843-1849. [PubMed: 20029048]

38. Jagasia M, Arora M, Flowers ME, et al. Risk factors for acute GVHD and survival after hematopoietic cell transplantation. Blood. 2012; 119:296-307. [PubMed: 22010102]

39. Sanz J, Boluda JC, Martin C, et al. Single-unit umbilical cord blood transplantation from unrelated donors in patients with hematological malignancy using busulfan, thiotepa, fludarabine and ATG as myeloablative conditioning regimen. Bone Marrow Transplant. 2012; 47:1287-1293. [PubMed: 22327127]

40. Brunstein CG, Weisdorf DJ, Defor T, et al. Marked increased risk of Epstein-Barr virus-related complications with the addition of anti-thymocyte globulin to a non-myeloablative conditioning prior to unrelated umbilical cord blood transplantation. Blood. 2006; 108:2874-2880. [PubMed: 16804113]

41. Cutler C, Stevenson K, Kim HT, et al. Double umbilical cord blood transplantation with reduced intensity conditioning and sirolimus-based GVHD prophylaxis. Bone Marrow Transplant. 2011; 46:659-667. [PubMed: 20697368]

42. Komanduri KV, St John LS, de Lima M, et al. Delayed immune reconstitution after cord blood transplantation is characterized by impaired thymopoiesis and late memory T-cell skewing. Blood. 2007; 110:4543-4551. [PubMed: 17671230]

43. Brunstein C, Barker JN, Weisdorf DJ, et al. Umbilical cord blood transplantation after nonmyeloablative conditioning: impact on transplant outcomes in 110 adults with hematological disease. Blood. 2007; 110:3064-3070. [PubMed: 17569820]

44. Soiffer RJ, Lerademacher J, Ho V, et al. Impact of immune modulation with anti-T-cell antibodies on the outcome of reduced-intensity allogeneic hematopoietic stem cell transplantation for hematologic malignancies. Blood. 2011; 117:6963-6970. [PubMed: 21464372]

45. Levine JE, Paczesny S, Sarantopoulos S. Clinical applications for biomarkers of acute and chronic graft-versus-host disease. Biol Blood Marrow Transplant. 2012; 18:S116-124. [PubMed: 22226094]

46. Harris AC, Ferrara JL, Braun TM, et al. Plasma biomarkers of lower gastrointestinal and liver acute GVHD. Blood. 2012; 119:2960-2963. [PubMed: 22286196]

47. Levine JE, Logan BR, Wu J, et al. Acute graft-versus-host disease biomarkers measured during therapy can predict treatment outcomes: a Blood and Marrow Transplant Clinical Trials Network study. Blood. 2012; 119:3854-3860. [PubMed: 22383800]

48. Delaney M, Cutler CS, Haspel RL, et al. High-resolution HLA matching in double-umbilical-cordblood reduced-intensity transplantation in adults. Transfusion. 2009; 49(5):995-1002. [PubMed: 19159415] 


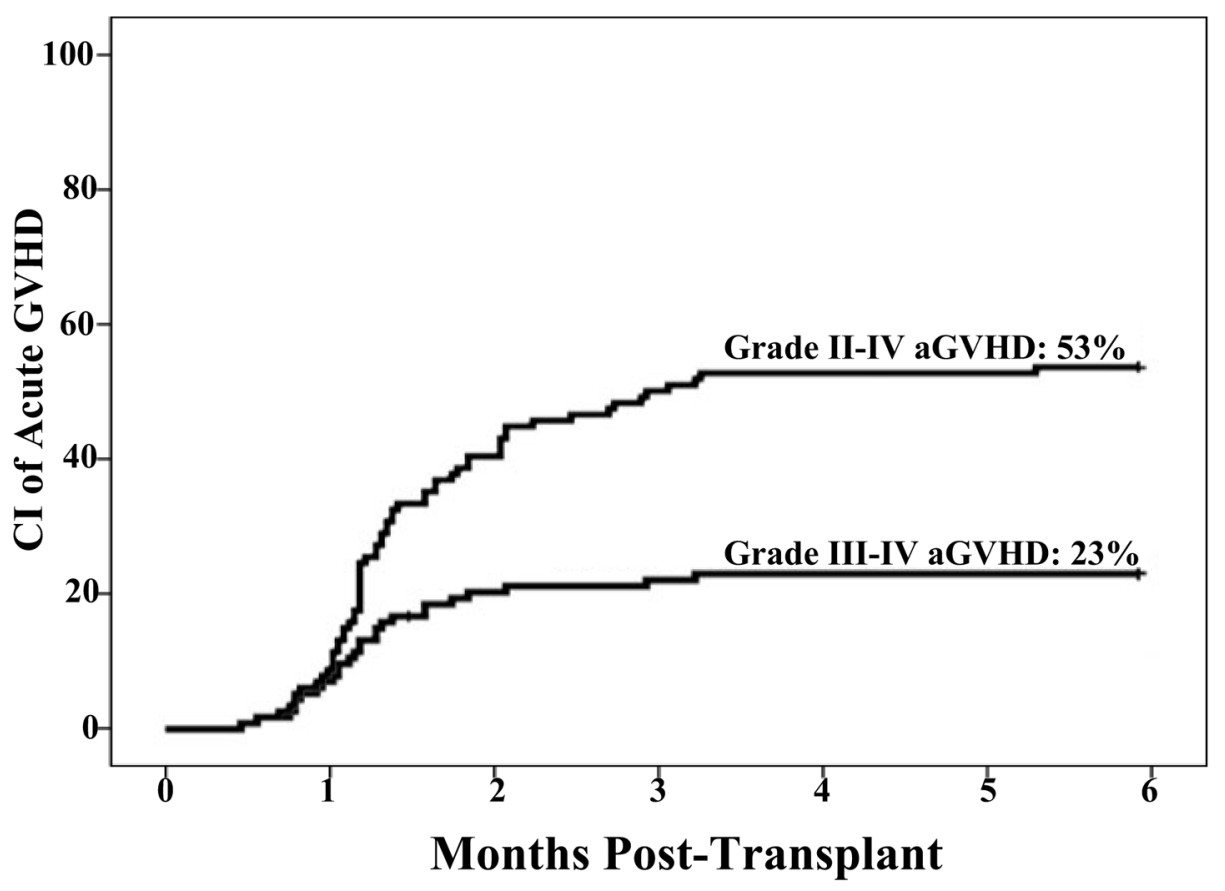

Figure 1. Cumulative incidence of grade II-IV and III-IV aGVHD at day 180

The incidence is reported at day 180 to include the 2 patients that had late onset aGVHD between days 100-180 after DCBT, and the 3 patients who developed aGVHD prior to day 100 but peaked at grade III-IV disease after day 100. These latter 3 patients, although grade II at onset, are included in the III-IV curve. 


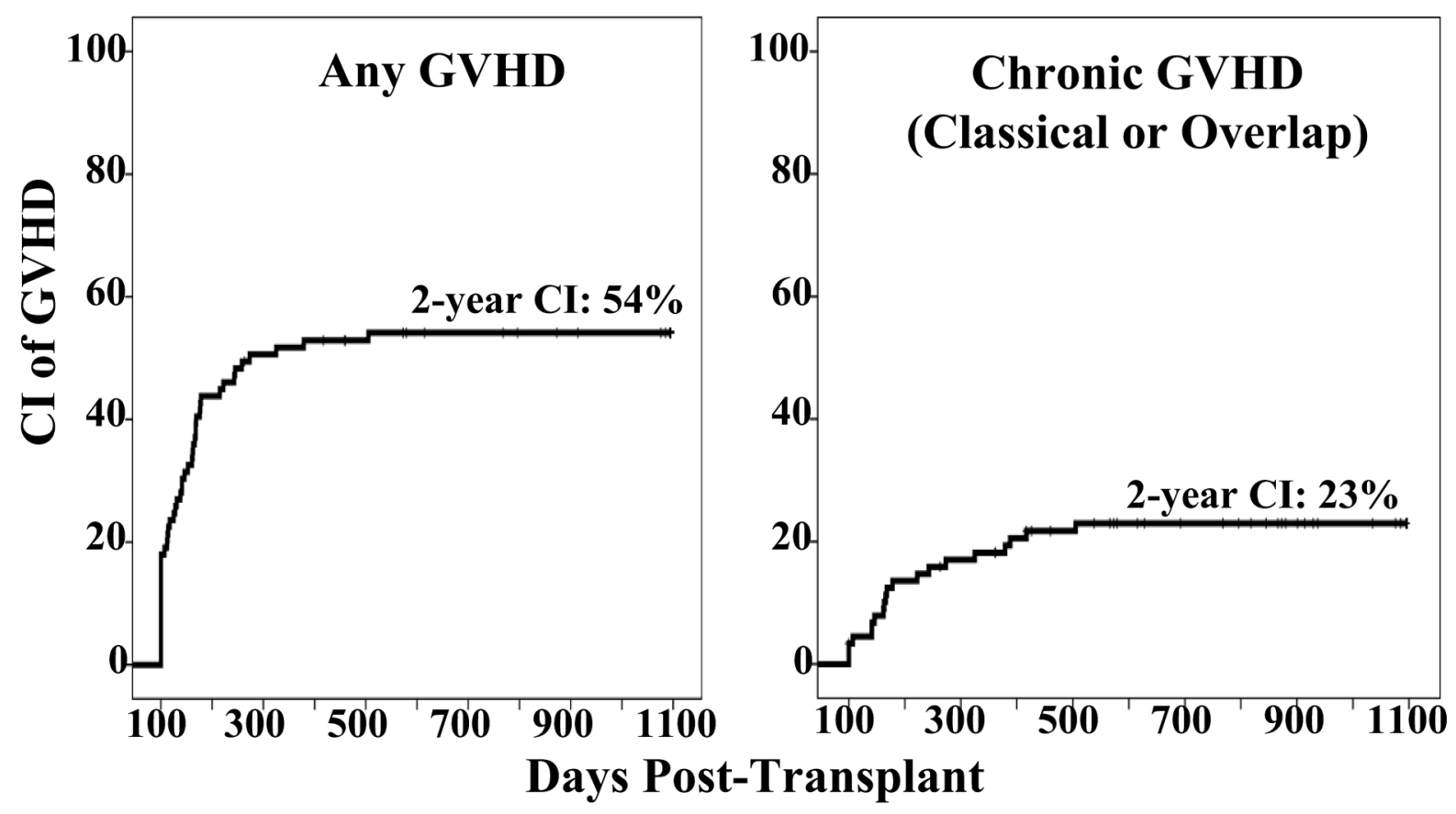

Figure 2. Cumulative incidence of GVHD in engrafted and disease-free at 100 days after transplantation

Figure 2A shows the cumulative incidence of any active GVHD after day 100, and 2B shows exclusively cGVHD (classical and overlap) after day 100 in this patient population. 


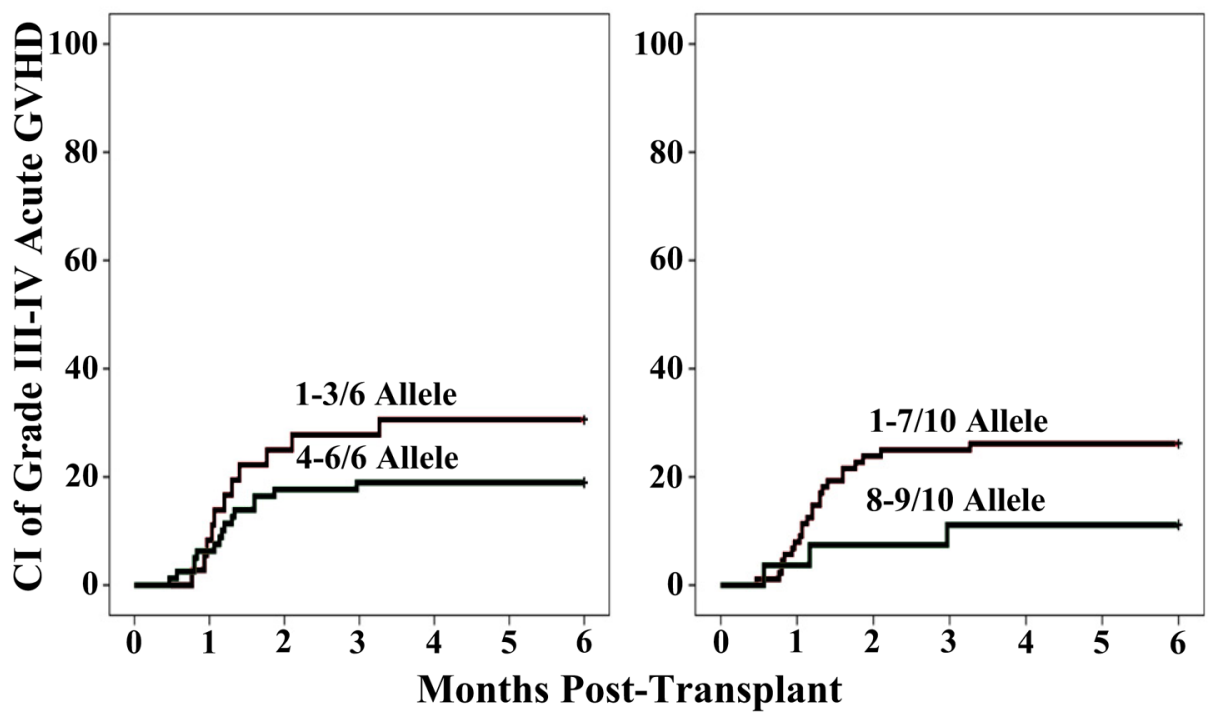

Figure 3.

Cumulative incidence of day 180 grade III-IV aGVHD by engrafting unit-recipient match at HLA-A,-B,-DRB1 (3A) and HLA-A,-B,-C,-DRB1,-DQ (3B) alleles. 
Table 1

Patient demographics $(\mathrm{n}=115)$.

\begin{tabular}{|c|c|}
\hline Characteristic & \\
\hline Median age (range) & 37 years $(0.9-69)$ \\
\hline $\mathbf{N}(\%)$ male/ female & $61(53 \%) / 54(47 \%)$ \\
\hline Median weight (range) & $66 \mathrm{~kg}(7-111)$ \\
\hline $\mathrm{N}(\%)$ recipient $\mathrm{CMV}$ sero-positive & $64(56 \%)$ \\
\hline $\mathbf{N}(\%)$ prior autologous transplantation & $16(14 \%)$ \\
\hline \multicolumn{2}{|l|}{$\mathrm{N}(\%)$ ancestry } \\
\hline European & $52(45 \%)$ \\
\hline Non-European & $63(55 \%)$ \\
\hline \multicolumn{2}{|l|}{$\mathbf{N}(\%)$ diagnosis } \\
\hline Acute leukemia (including ABL) & $67(58 \%)$ \\
\hline MDS/ CML/ Other myeloproliferative disease & $6(5 \%)$ \\
\hline NHL/ HD/ CLL & $42(37 \%)$ \\
\hline \multicolumn{2}{|l|}{$\mathbf{N}(\%)$ disease risk $(\%)^{*}$} \\
\hline Standard & $21(18 \%)$ \\
\hline High & $94(82 \%)$ \\
\hline \multicolumn{2}{|l|}{ N (\%) high dose myeloablative conditioning } \\
\hline Cy 120/ Flu 75/ TBI 1320-1375 cGy & $43(37 \%)$ \\
\hline Clo 100-150/ Mel 140/ Thio 10 & $11(10 \%)$ \\
\hline Thio 10/ Flu 125/ TBI 1375 cGy & $2(2 \%)$ \\
\hline \multicolumn{2}{|l|}{$\mathrm{N}(\%)$ reduced intensity conditioning } \\
\hline Flu 150/ Cy 50/ Thio 10/ TBI 400 cGy & $25(22 \%)$ \\
\hline Mel 140/ Flu 150 & $7(6 \%)$ \\
\hline \multicolumn{2}{|l|}{$\mathrm{N}(\%)$ non-myeloablative conditioning } \\
\hline Flu 150/ Cy 50/ TBI 200 cGy & $27(23 \%)$ \\
\hline
\end{tabular}

Abbreviations: N, number; Kg, kilogram; CMV, cytomegalovirus; ABL, acute biphenotypic leukemia; MDS, myelodysplastic syndrome; CML, chronic myeloid leukemia; NHL, non-Hodgkin's lymphoma; HD, Hodgkin's Disease; CLL, chronic lymphocytic leukemia; Cy, cyclophosphamide; Flu, fludarabine; TBI, total body irradiation; Clo, clofarabine; Mel, melphalan; Thio, thiotepa.

* Standard risk disease for AML and ALL was defined as CR1 without high-risk cytogenetics or high-risk molecular abnormalities, de novo MDS with an International Prognostic Scoring System score < 2, chronic myelogenous leukemia in first chronic phase, and chemotherapy sensitive lymphoma in less than second relapse for aggressive histologies of less than third relapse for indolent disease without prior autologous transplantation. All remaining patients were considered high-risk. 
Table 2

Graft characteristics ( $\mathrm{n}=115$ double-unit grafts, 230 units).

\begin{tabular}{|c|c|}
\hline Characteristic & \\
\hline Infused cell dose & $\underline{\text { Median (range) }}$ \\
\hline $\mathrm{TNC} \times 10^{7} / \mathrm{kg}$ & \\
\hline Larger unit / Smaller unit & $2.7(1.4-12.8) / 2.0(0.9-7.1)$ \\
\hline $\mathrm{CD34}+\times 10^{5} / \mathrm{kg}$ & \\
\hline Larger unit / Smaller unit & $1.2(0.3-7.0) / 0.7(0.1-2.1)$ \\
\hline $\mathrm{CD} 3+\times 10^{6} / \mathrm{kg}$ & \\
\hline Larger unit / Smaller unit & $4.4(1.3-15.0) / 3.1(0.3-10.6)$ \\
\hline \multicolumn{2}{|l|}{ Unit-recipient HLA-match } \\
\hline$A$ and $-B$ antigen, -DRB1 allele & $\underline{\mathbf{N}(\%)}$ \\
\hline $6 / 6$ & $11(5 \%)$ \\
\hline $5 / 6$ & $121(52.5 \%)$ \\
\hline $4 / 6$ & $98(42.5 \%)$ \\
\hline \multicolumn{2}{|l|}{ A, -B, -DRB1 allele } \\
\hline $6 / 6$ & $7(3 \%)$ \\
\hline $5 / 6$ & $63(27 \%)$ \\
\hline $4 / 6$ & $73(32 \%)$ \\
\hline $3 / 6$ & $62(27 \%)$ \\
\hline $2 / 6$ & $23(10 \%)$ \\
\hline \multirow[t]{2}{*}{$1 / 6$} & $2(1 \%)$ \\
\hline & $\underline{\text { Median (range) }}$ \\
\hline A, -B,-DRB1 allele & $4 / 6(1-6 / 6)$ \\
\hline A, -B, -C, -DRB1, -DQ allele & $6 / 10(2-9 / 10)$ \\
\hline Unit-unit HLA-match & Median (range) \\
\hline$A$ and $-B$ antigen, -DRB1 allele & $4 / 6(2-6 / 6)$ \\
\hline A, -B, -DRB1 allele & $4 / 6(0-6 / 6)$ \\
\hline A, -B, -C, -DRB1, -DQ allele & $5 / 10(0-10 / 10)$ \\
\hline
\end{tabular}

Abbreviations: N, number; HLA, human leukocyte antigen. 


\section{Table 3}

aGVHD therapy responses in patients treated with either systemic corticosteroids or budesonide.

\begin{tabular}{|l|c|c|}
\hline & Systemic Corticosteroids (N = 29) & Budesonide Alone (N = 27) \\
\hline Median time to GVHD onset & 34 days (range 14-89) & 54 days (range 31-161) \\
\hline N (\%) organ involvement & & \\
Skin alone & $5(17 \%)$ & 0 \\
GI & $23(79 \%)$ & $27(100 \%)$ \\
Skin/ GI & $12(52 \%)$ & $12(44 \%)$ \\
GI alone & $3(13 \%)$ & $14(52 \%)$ \\
GI/ Liver & $4(17 \%)$ & $1(4 \%)$ \\
GI/ Liver/Skin & $4(17 \%)$ & 0 \\
Liver alone & $1(3 \%)$ & $27(100 \%)$ \\
\hline N $(\%)$ aGVHD grade at treatment onset & & 0 \\
II & $8(28 \%)$ & 0 \\
III & $16(55 \%)$ & $10(37 \%)$ \\
IV & $5(17 \%)$ & $13(48 \%)$ \\
\hline N (\%) treatment response at day 28 of therapy & & $4(15 \%)$ \\
CR & $11(38 \%)$ & \\
PR & $12(41 \%)$ & \\
< PR & $6(21 \%)$ & \\
\hline
\end{tabular}

Abbreviations: N, number; GI, gastro-intestinal tract; aGVHD, acute graft-versus-host disease; CR, complete response; PR, partial response. 


\section{Table 4}

Summary of GVHD syndromes after day $100(n=48$ patients of 89 engrafted and disease-free at day 100$)$.

\begin{tabular}{|c|c|c|}
\hline GVHD Syndrome & $\mathbf{N}(\%)^{*}$ & Severity \\
\hline aGVHD & $31(64.5 \%)$ & \\
\hline Persistent or recurrent & 29 & 21 grade II \\
\hline Late onset II-IV aGVHD (days 100-180) & 2 & 10 grade III-IV \\
\hline Classical chronic & $10(21 \%)$ & \\
\hline Interrupted & 4 & 9 mild \\
\hline De novo & 6 & 5 moderate \\
\hline Overlap syndrome ${ }^{* *}$ & $7(14.5 \%)$ & 3 severe \\
\hline
\end{tabular}

Abbreviations: GVHD, graft-versus-host disease; N, number; aGVHD, acute graft-versus-host disease.

* Percentages reflect proportion of the 48 patients affected with GVHD after day 100.

$$
\text { *** } 3
$$

3 patients with persistent aGVHD subsequently evolved to overlap syndrome ( 1 mild, 2 severe). Thus, there were a total of 10 patients who developed overlap syndrome during the study period. 
Table 5

Multivariate analysis of variables potentially associated with day 180 grade III-IV aGVHD incidence $(\mathrm{n}=$ 115).

\begin{tabular}{|c|c|c|}
\hline Patient and Graft Characteristics & Hazard Ratio ${ }^{*}$ & $P$ value \\
\hline \multicolumn{3}{|l|}{ Age (years) } \\
\hline $0-15(\mathrm{n}=23)$ & Reference & \\
\hline$\geq 16(\mathrm{n}=92)$ & $0.920(0.358-2.36)$ & 0.862 \\
\hline \multicolumn{3}{|l|}{ CMV serostatus } \\
\hline Sero-negative $(\mathrm{n}=51)$ & Reference & \\
\hline Sero-positive $(n=64)$ & $0.570(0.255-1.275)$ & 0.171 \\
\hline \multicolumn{3}{|l|}{ Engrafting unit $\mathrm{TNC} \times 10^{7} / \mathrm{kg}$} \\
\hline$<3.0(\mathrm{n}=94)$ & Reference & \\
\hline$\geq 3.0(\mathrm{n}=21)$ & $2.040(0.802-5.217)$ & 0.134 \\
\hline \multicolumn{3}{|l|}{ Engrafting unit-recipient 6 allele HLA-match } \\
\hline $1-3 / 6(n=36)$ & Reference & \\
\hline $4-6 / 6(n=79)$ & $0.385(0.162-0.915)$ & 0.031 \\
\hline \multicolumn{3}{|l|}{ Unit-unit 6 allele HLA-match } \\
\hline $0-4 / 6(n=88)$ & Reference & \\
\hline $5-6 / 6(n=27)$ & $2.204(0.866-5.609)$ & 0.097 \\
\hline
\end{tabular}

Abbreviations: aGVHD, acute graft-versus-host disease; CMV, cytomegalovirus; HLA, human-leukocyte antigen; TNC, total nucleated cell; kg, kilogram.

Includes $95 \%$ confidence interval. 